\title{
Collimation method that uses spiral gratings and Talbot interferometry
}

\author{
Chon-Wen Chang and Der-Chin Su \\ Institute of Electro-Optical Engineering, National Chiao-Tung University, 1001 Ta-hsueh Road, Hsin-Chu, Taiwan, China
}

Received July 8, 1991

\begin{abstract}
The tested light is projected onto an arrangement composed of two special spiral gratings, in which the second spiral grating is positioned at the Talbot image of the first one. From the shapes of the resultant moiré fringes, the quality of collimation of the tested light can be easily checked.
\end{abstract}

Collimated monochromatic light is often used in optical metrology. The error of collimation will influence the resulting measurement and reduce the accuracy of measurement. Therefore an easy and simple method to check the quality of collimation of the light is important. Several papers ${ }^{1-5}$ for checking collimation of the light beam have been published that are based on the Talbot effect and moiré technique. Although these studies used simple and easily adjustable setups, the resultant moiré fringes are in linear distribution in spite of the convergence of the light because they all used linear gratings. Hence it is not easy to distinguish them clearly.

In this Letter a collimation method that uses two special spiral gratings is proposed. It is also based on Talbot interferometry. The resultant moiré fringes are distributed radially for collimated light and spirally for noncollimated. light. Moreover it is easy to tell whether the light source is inside or outside the focus according to the direction of the spiral distribution.

The optical arrangement is shown in Fig. 1. Monochromatic light is incident upon the first spiral grating SG1 and is then projected onto the second spiral grating SG2. SG1 and SG2 are two special spiral gratings, as shown in Figs. 2 and 3. They have the same radial period $p$ but are in different directions, and SG2 is positioned at the Talbot image of SG1. For simplicity, let the amplitude transmittances of SG1 and SG2 be written as

$$
\begin{aligned}
& t_{1}(r, \theta)=\frac{1}{2}+\frac{1}{2} \cos \left(\frac{2 \pi r}{p}-4 \theta\right), \\
& t_{2}(r, \theta)=\frac{1}{2}+\frac{1}{2} \cos \left(\frac{2 \pi r}{p}+4 \theta\right)
\end{aligned}
$$

respectively. Since SG2 is positioned at the Talbot image of SG1, the wave field on SG2 is given by

$$
t_{1}^{\prime}(r, \theta)=\frac{1}{2}+\frac{1}{2} \cos \left(\frac{2 \pi r}{p^{\prime}}-4 \theta\right)
$$

where $p^{\prime}$ is the radial period of the projection of SG1 onto SG2. According to the Talbot effect, it is obvious that $p^{\prime}=p$ for collimated light and that $p^{\prime} \neq p$ for noncollimated light. Then the intensity on the observation plane OP located just behind SG2 can be written as

$$
I(r, \theta)=\left|t_{1}^{\prime}(r, \theta) t_{2}(r, \theta)\right|^{2}
$$

If Eqs. (2), (3), and (4) are combined, the intensity distribution $I_{M}(r, \theta)$ of the resultant moiré fringes is given in the form of

$$
I_{M}(r, \theta) \sim \cos \left(\frac{2 \pi r}{p^{\prime}}-\frac{2 \pi r}{p}-8 \theta\right) .
$$

From relation (5) it can be seen that there are eight sets of moire fringes, when $\theta$ is from 0 to $2 \pi$. The shapes of these eight sets of moiré fringes are determined by the following equation:

$$
\frac{2 \pi r}{p^{\prime}}-\frac{2 \pi r}{p}-8 \theta=2 N^{\prime} \pi, \quad N^{\prime}=0,1, \ldots 7 .
$$

For collimated light since $p^{\prime}=p$, Eq. (6) can be rewritten as

$$
\theta=-\frac{1}{4} N^{\prime} \pi, \quad N^{\prime}=0,1, \ldots 7 .
$$

The shapes of the moire fringes are radial.

For noncollimated light, since $p^{\prime} \neq p$, Eq. (6) can be rewritten as
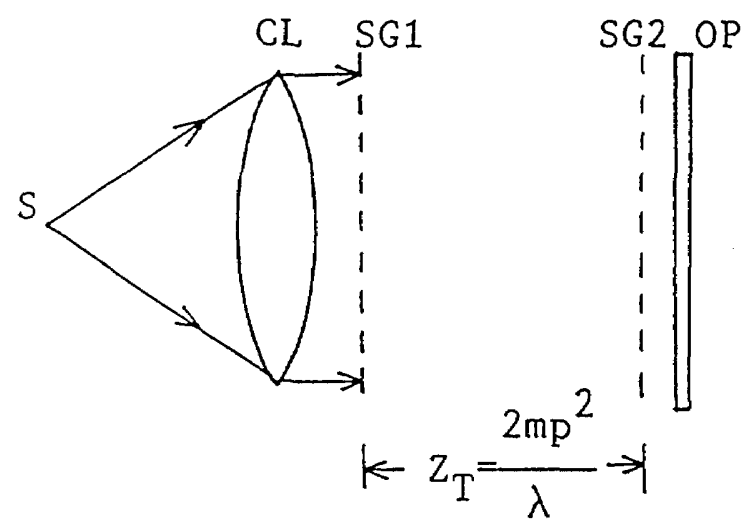

Fig. 1. Optical arrangement for measuring the collimated light. CL, collimating lens; SG's, special spiral gratings; OP, observation plane. 


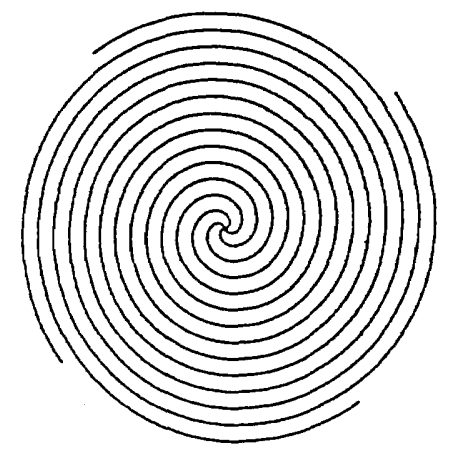

Fig. 2. Diagram of SG1.

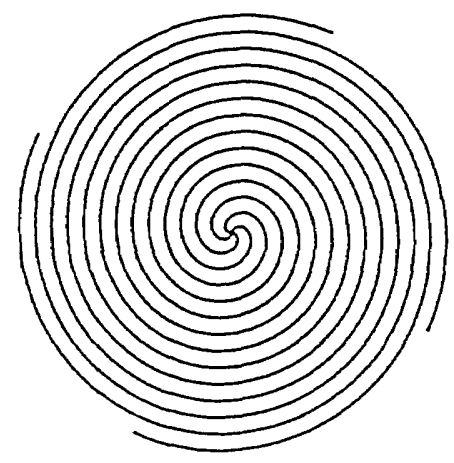

Fig. 3. Diagram of SG2.

$r=\frac{p^{\prime} p}{p-p^{\prime}} \frac{4}{\pi}\left(\theta+\frac{\pi}{4} N^{\prime}\right)=k\left(\theta+\frac{\pi}{4} N^{\prime}\right)$,

$$
N^{\prime}=0,1, \ldots 7 \text {. }
$$

From Eq. (8) it is easily understood that the shapes of the moiré fringes are spiral.

For divergent light, we have $p^{\prime}>p$, then $k<0$. The spiral moiré fringes are clockwise. Because the light source is inside the focus, the collimated lens CL should be moved away from the light source.

For convergent light, we have $p^{\prime}<p$, then $k>0$. The spiral moiré fringes are counterclockwise. Because the light source is outside the focus, the collimated lens CL should be moved toward the light source.

In order to demonstrate the feasibility of this method, we use two self-made spiral gratings with a radial period of $0.4 \mathrm{~mm} /$ line to check the collimation of the light. SG2 is positioned at the first Talbot image of SG1, that is, $m=1$. A He-Ne laser with a wavelength $\lambda=632.8 \mathrm{~nm}$ is used in this experiment. The resultant moiré fringes are shown in Fig. 4 for (a) quasi-collimated light, (b) divergent light, and (c) convergent light.

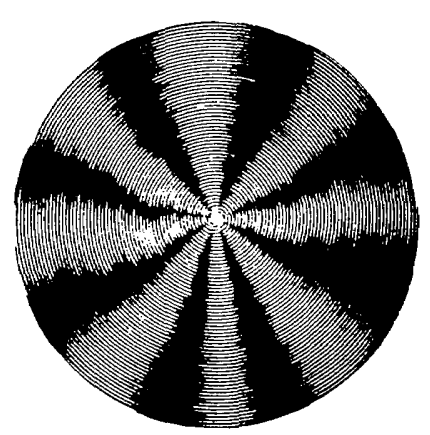

(a)

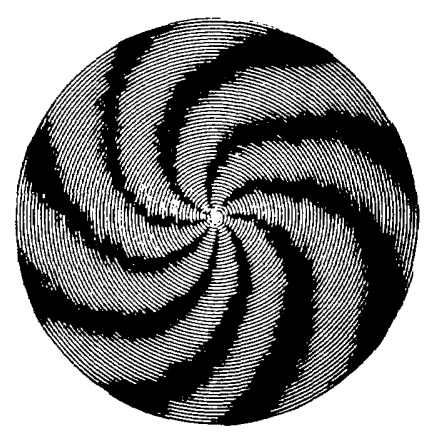

(b)

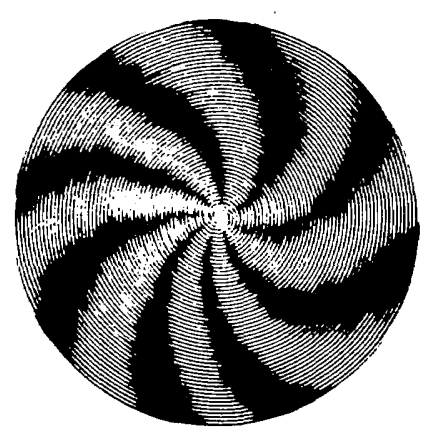

(c)

Fig. 4. Moiré fringes for (a) quasi-collimated light, (b) divergent light, and (c) convergent light.

This study was supported in part by the National Science Council, Republic of China, under contract number NSC80-0417-E009-16.

\section{References}

1. D. E. Silva, Appl. Opt. 10, 1980 (1971).

2. J. C. Fouere and D. Malacara, Appl. Opt. 13, 1322 (1974).

3. S. Yokozeki, K. Patorski, and K. Ohnishi, Opt. Commun. 14, 401 (1975).

4. K. Patorski, S. Yokozeki, and T. Suzuki, Appl. Opt. 15, 1234 (1976).

5. M. P. Kothiyal and R. S. Sirohi, Appl. Opt. 26, 4056 (1987). 